\title{
Ensure an original and safe "Fail-to-Open" mode in planar and trench power SiC MOSFET devices in extreme short-circuit operation
}

\author{
F. Boige ${ }^{a,}$, F. Richardeau ${ }^{a}$, S. Lefebvre ${ }^{b}$, J.-M. Blaquière $^{\mathrm{a}}$, G. Guibaud $^{\mathrm{c}}$, A. Bourennane $^{\mathrm{d}}$ \\ ${ }^{\text {a }}$ LAPLACE, University of Toulouse, CNRS, INPT, UPS, France. \\ ${ }^{\mathrm{b}}$ SATIE, CNAM, CNRS, ENS Paris-Saclay, Cachan, France. \\ ${ }^{\mathrm{c}}$ THALES Communications \& Security, ITEC Lab, CNES, Toulouse, France. \\ ${ }^{d}$ LAAS-CNRS, University of Toulouse, CNRS, Toulouse, France.
}

\begin{abstract}
The purpose of this paper is to present a complete experimentation of the two failure modes in competition that can appear during short-circuit (SC) fault operation of single-chip $1,2 \mathrm{kV} \mathrm{SiC}$ MOSFETs from different manufacturers including planar and trench-gate structures, well-known or recent devices. Ruggedness and selective failure modes are identified in relation with the power density dissipated by the chip and the simulated 1D-thermal junction. Finally, the chips of the devices which failed in a "fail-to-open" mode have been studied in order to find the physical reasons of this original and unusual fail-safe mode.
\end{abstract}

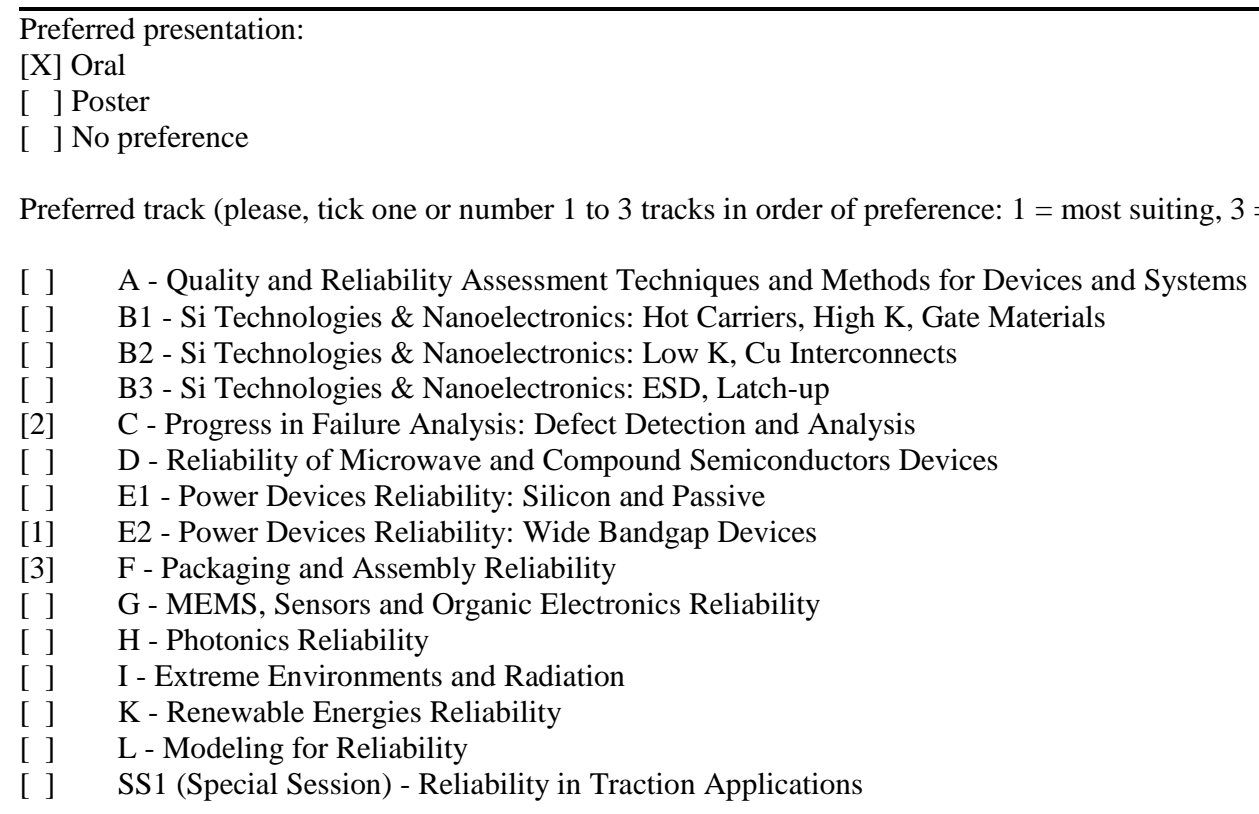

\section{Corresponding author.}

boige@laplace.univ-tlse.fr

Tel: +33534322407 
Ensure an original and safe "Fail-to-Open" mode in planar and trench power SiC MOSFET devices in extreme short-circuit operation

F. Boige ${ }^{a, *}$, F. Richardeau ${ }^{\text {a }}$, S. Lefebvre ${ }^{b}$, J.-M. Blaquière $^{\mathrm{a}}{ }^{\text {G. G. Guibaud }}{ }^{\mathrm{c}}$, A. Bourennane $^{\mathrm{d}}$ 


\section{Introduction}

Recently, several research efforts demonstrate that ruggedness of $\mathrm{SiC}$ power MOSFETs during short-circuit (SC) is, for most of devices, much lower than silicon ( $\mathrm{Si})$ devices, with similar rating [1]. Moreover, in $\mathrm{SiC}$ MOSFET, two different chip failure modes that may appear have been pointed out [1], [2]. The first one is the classical "fail-to-short" (FTS) mode which means that the device present a permanent small residual resistance between the drain and the source electrodes of the chip after the short-circuit operation [3]. This failure mode is the only one observed today with silicon devices. The second one, is a permanent short-circuit between the gate and the source electrodes of the chip leading to the turn off of the channel which means that the device is still holding the bus voltage and cannot be turned on again in extreme operation. This mode is called "fail-to-open" (FTO). The FTO is clearly promising for safety application: the device is automatically protected permitting the freewheeling of the load current through its body diode. However, depending on the SiC MOSFET technology and/or the conditions of use, only one failure mode can prevail upon the other [2]. Authors have divided this article into three main parts. In section 2 are presented the experimental setup and the measurement methodology. Section 3 investigates the validity domain of the FTS and FTO failure modes. In particular, using a previously developed transient thermal model [4] this section links the temperature estimation to the dissipated power density into the device but also presents physical analysis of the chips after failure. In section 4 , a better physical understanding of the FTO consolidation mode is proposed in order to inhibit the FTS concurrent mode.

\section{Experimental setup and measurement protocol}

Type 1 short-circuit tests were performed on nine discrete-types $\mathrm{SiC}$ MOSFETS in TO247 case from five different manufacturers (noted $\mathrm{A}$ to $\mathrm{E}$ ) including different generation-type of devices (noted 1 for the oldest to 3 for the most recent). Main electrical characteristics are summarised in Table 1.
I. Electrical characteristics of the tested devices and their

\begin{tabular}{|c|c|c|c|c|c|c|c|c|c|}
\hline Device & A1 & A2 & A3 & B1 & B2 & B3 & $\mathrm{C} 1$ & D1 & E1 \\
\hline $\mathrm{S}_{\text {chip }}\left(\mathrm{mm}^{2}\right)$ & 16.5 & 10.4 & 6.48 & 13.6 & 12.4 & 5.3 & 16.7 & 11.8 & 10.6 \\
\hline $\mathrm{V}_{\mathrm{Dss}}(\mathrm{V})$ & 1200 & 1200 & 900 & 1200 & 1200 & 1200 & 1200 & 1200 & 1200 \\
\hline $\mathrm{I}_{\mathrm{D}} @ 25^{\circ} \mathrm{C}(\mathrm{A})$ & 42 & 36 & 36 & 26 & 40 & 31 & 41 & $*$ & 25 \\
\hline $\mathrm{V}_{\mathrm{GSnom}(\mathrm{on} / \mathrm{of})}(\mathrm{V})$ & $20 /-5$ & $20 /-5$ & $15 /-3$ & $18 /-5$ & $18 /-5$ & $18 /-5$ & $20 /-5$ & $15 /-3$ & $20 /-5$ \\
\hline $\begin{array}{l}\mathrm{R}_{\mathrm{DS}(\mathrm{on})}(\mathrm{m} \Omega) \\
\text { (a) } \mathrm{V}_{\mathrm{GSnom}}\end{array}$ & 80 & 80 & 65 & 90 & 80 & 80 & 80 & 80 & 80 \\
\hline Technology & planar & planar & planar & $\begin{array}{l}\text { shield- } \\
\text { planar }\end{array}$ & $\begin{array}{l}\text { shield- } \\
\text { planar }\end{array}$ & trench & * & trench & * \\
\hline
\end{tabular}

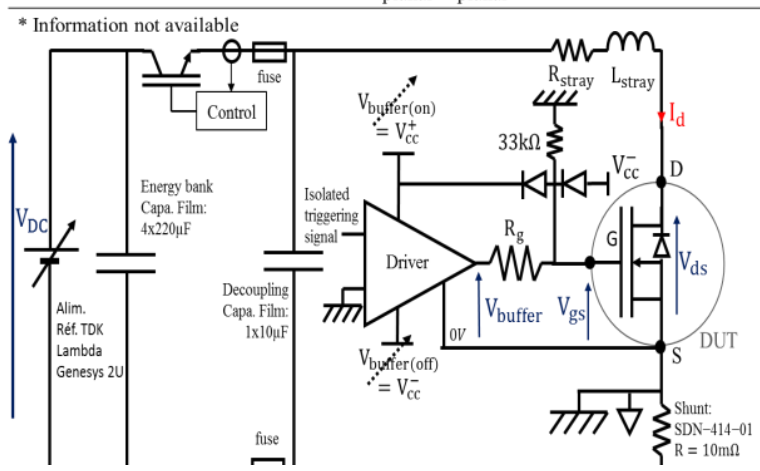

Fig. 1. Type 1 short-circuit test schematic.

Tektronix DPO4014B oscilloscope $(1 \mathrm{GHz}-$ $5 \mathrm{GS} / \mathrm{s})$ with 2 Tektronix TPP1000 (300V $1 \mathrm{GHz}$ $3.9 \mathrm{pF})$ and 1 TPP0850 (800MHz $1000 \mathrm{~V} \quad 1.8 \mathrm{pF})$ voltage probes are used for the measurements of $\mathrm{V}_{\text {buffer, }}, \mathrm{V}_{\mathrm{gs}}$ and $\mathrm{V}_{\mathrm{ds}}$ respectively. Drain current is measured using an aselfic coaxial shunt (propagation times between current and voltage measurements have been compensated). The stray inductance is estimated to a value close to L_stray $=52 \mathrm{nH}$

The aim of the measurements is testing the devices in an extreme type 1 short-circuit until the die destruction. Emphasis has been put on finding the validity domains of

The device under test (DUT) is turned-on across a voltage source and through a polypropylenecapacitors tank providing the high- current pulse. The test bench allows to fix the DUT to the test bench near the case - deleting some parasitic influences of pins. A high current IGBT mounted into the test bench is used as a fast electronic breaker (threshold current $\mathrm{I}_{\mathrm{th}}=500 \mathrm{~A}$, detection time $<100 \mathrm{~ns}$ and propagation + blocking time $<1 \mu \mathrm{s}$ ) to keep the DUT from further damage after short-circuit failure. CMS fuses are used for capacitor safety in last resort. Fig. 1 shows the schematic of the proposed experimental setup. The gate turn-on/turn-off bias are summarised in Table I.

\section{Experimental results}

\subsection{Focus on the device A2}




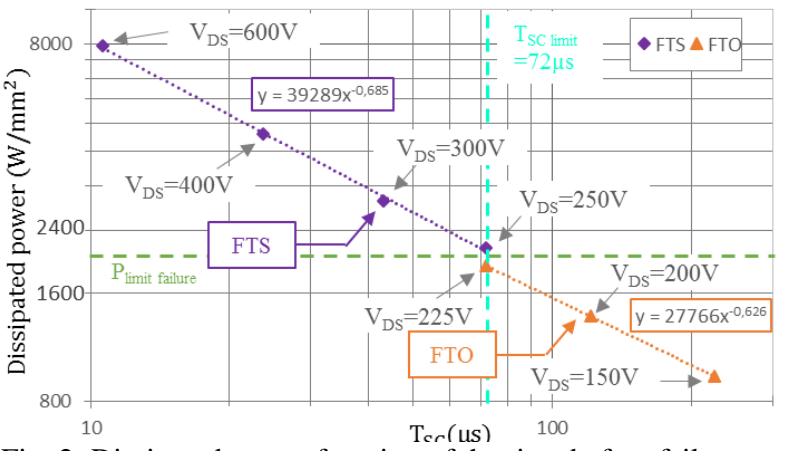

Fig. 2: Dissipated power function of the time before failure for the device $\mathrm{A} 2$. $\mathrm{V}_{\text {buffer( }(\mathrm{ON} / \mathrm{OFF})}=+20 \mathrm{~V} /-5 \mathrm{~V}, \mathrm{R}_{\mathrm{G}}=47 \Omega$.

In the literature, it appears that the device A2 presents the two kind of failure mode [2]. In order to find the limits between the two modes an extensive destructive study has been carried out. In Fig. 2, each point represents a device tested at a fixed drain bias until the destruction occurs. The average density losses dissipated in the chip is calculated by the total energy dissipated divided by the short-circuit duration until failure $\left(\mathrm{T}_{\mathrm{SC}}\right)$ and the die area. The FTO mode only appears under $\mathrm{V}_{\mathrm{DS}}=250 \mathrm{~V}$ and after $72 \mu \mathrm{s}$ of short-circuit duration. In contrast, the FTS mode appears from $250 \mathrm{~V}$ to the maximum testing voltage $(600 \mathrm{~V})$ and $\mathrm{T}_{\mathrm{SC}}$ lower than $72 \mu \mathrm{s}$. It seems that, to appear, the FTO mode probably needs a lower thermal dissipation and therefore a slower transient temperature rise in order to consolidate the failure mechanism. To confirm this hypothesis, a previously developed 1D transient thermal model has been used to estimate the junction temperature. Results are presented in Fig. 3 [4]. Indeed, in short-circuit operation, the heat generation is very great and last a very short time less than the die thermal time constant. Therefore, the die thermal model can be simplified to a 1D heat propagation line with a punctual power source [5]. For study purposes, dependence with temperature of the thermal conductivity and the specific heat of $4 \mathrm{H}-\mathrm{SiC}$ and aluminum have been considered [6], [7]. The phase transition energy of the die aluminum top metal has also been modeled, and its effect can be shown in Fig.3. Note that the temperature estimated by the model cannot be calibrated today by direct or indirect thermal measurements on the die so the temperature estimation must be considered with caution.

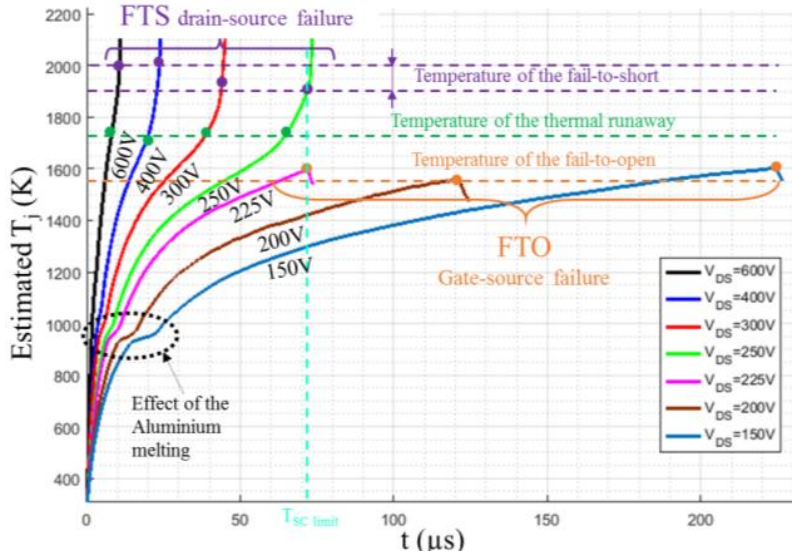

Fig. 3: Estimated maximum junction temperature for different drain bias for the device A2 during SC operations.
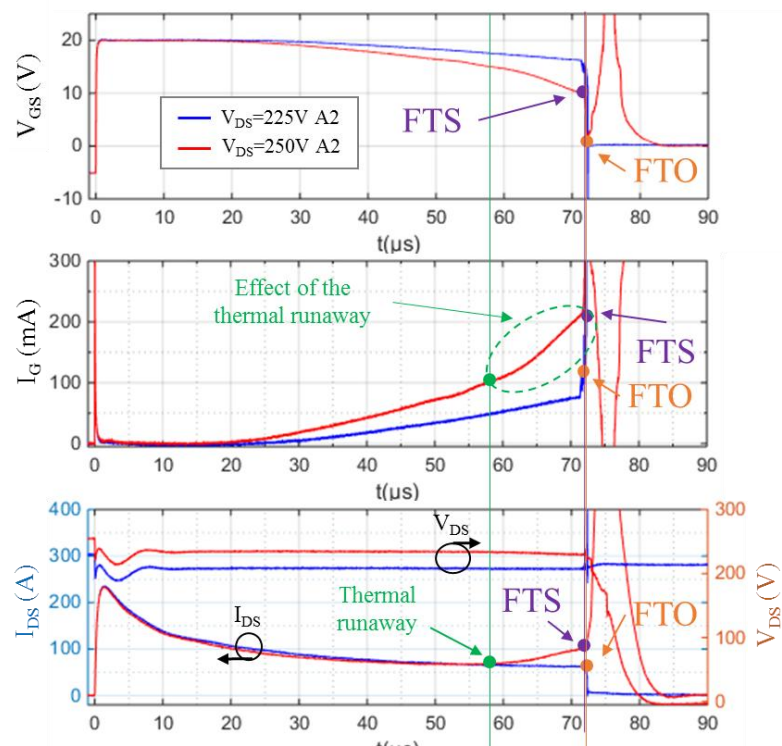

Fig. 4: Experimental waveforms during short-circuit of the device $\mathrm{A} 2$. $\left(\mathrm{V}_{\mathrm{DS}}=225 / 250 \mathrm{~V} ; \mathrm{V}_{\text {buffer(on/off) }}=\right.$ $+20 \mathrm{~V} /-5 \mathrm{~V} ; \mathrm{T}_{\text {case }}=25^{\circ} \mathrm{C} ; \mathrm{R}_{\mathrm{G}}=47 \Omega$ ).

The thermal model results show that the failures, for different drain bias, always happen for a maximum temperature close to $1600 \mathrm{~K}$ in FTO mode and close to $1900 \mathrm{~K}$ in FTS mode. The differences in the two failure modes lie in the temperature rises, as suggested in [2]. Indeed, in the case of a FTS mode, the temperature rise reaches $1600 \mathrm{~K}$ but in a too short time in order the physical mechanism of the FTO to take place on a consolidated basis. Instead, the temperature still rises to $1700 \mathrm{~K}$, the temperature value which seems to be the threshold inducing the thermally generated leakage current inside the chip to start to increase and/or to start to turn-on the 
intrinsic parasitic BJT. Up to this point, the drain current starts to increase even more leading to more power dissipation into the chip as well as a faster rise in temperature until the destruction in FTS mode (latch-up or thermal runaway).

The difference between the waveforms of the two failure modes are depicted in fig.4. For the waveforms obtained at $\mathrm{V}_{\mathrm{DS}}=250 \mathrm{~V}$, the drain current generation is clearly noticeable. Moreover, still in Fig. 4, a strong gate current flows through the gate of the device after about $20 \mu$ s of short-circuit duration. This phenomenon has already been discussed in [8] and appears for all the tested devices in short-circuit operation. This current is damaging the device in the gate region but cannot be directly correlated to one failure mode. Moreover, this gate current is assumed to be thermionic emission [9], this physical mechanism is strongly related to the temperature and the electric field through the oxide so it is an image of the temperature in the oxide region.

\subsection{Overview of the devices}

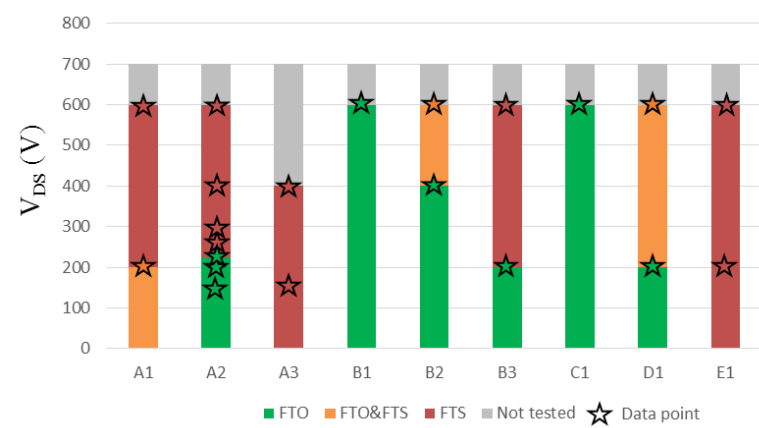

Fig. 5. Summary of the failure modes of the devices under test for different drain bias

The other presented devices have been also tested in order to find their failure modes and, in the case where the two failure modes are in competition, the time $\mathrm{T}_{\mathrm{SC}}$ which separate the two failure modes. Generally, the devices were tested at two voltage bias, $\mathrm{V}_{\mathrm{DS}}=600 \mathrm{~V}$ and $200 \mathrm{~V}$ with an important derating.

As presented on Fig. 5, on nine devices under test, six of them presented the FTO failure mode, four at low drain bias (A2, B2, B3, D1) and two at $\mathrm{V}_{\mathrm{DS}}=600 \mathrm{~V}(\mathrm{~B} 1 \& \mathrm{C} 1)$. The two latest are clearly the most interesting devices because their intrinsic FTO mode can be obtained even if these devices are used in nominal condition. B1 being obsolete, so device $\mathrm{C} 1$ is the most promising device for future fail-safe applications. The waveforms during destructive SC of device $\mathrm{C} 1$ are displayed in Fig. 6.
The thermal model has been adapted for each device (die area). Some of the results are displayed in Fig. 7. Failure temperature in FTO mode are estimated to $1500 \mathrm{~K}$ and $1700 \mathrm{~K}$ for devices $\mathrm{C} 1$ and $\mathrm{B} 2$ respectively. These two temperatures are similar to the failure temperature in FTO estimated for device A2. These results tend to prove the uniqueness of the failure mechanism in such failure mode.
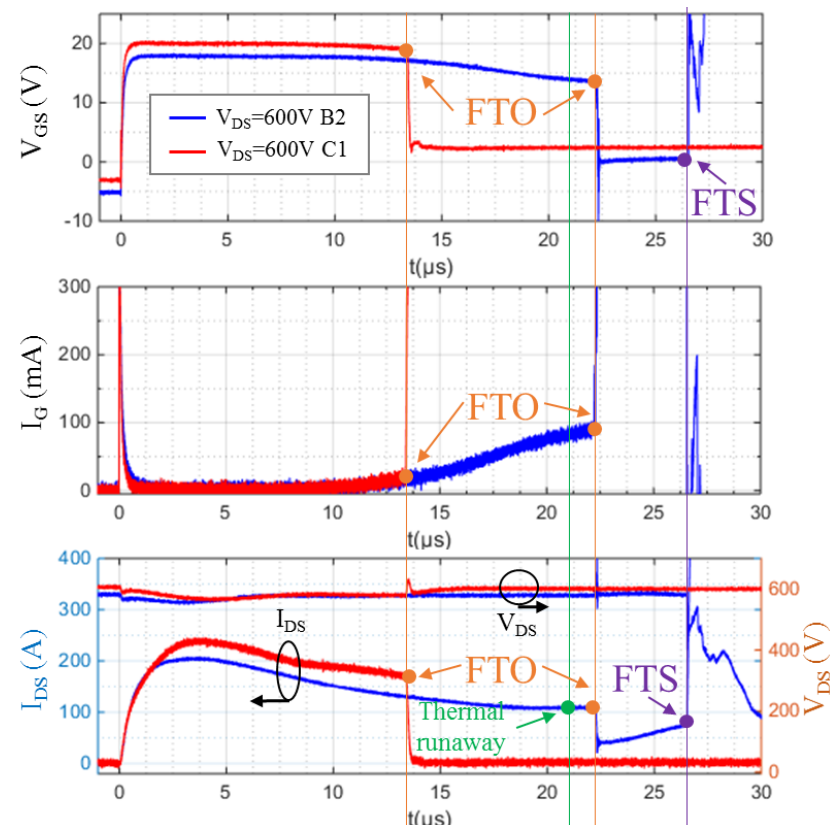

Fig. 6: Experimental waveforms during short-circuit for device $\mathrm{B} 2$ and $\mathrm{C} 1$. ( $\mathrm{V}_{\mathrm{DS}}=600 \mathrm{~V} ; \mathrm{V}_{\text {buffer}(\text { on } / \mathrm{off})}=$ $+20 \mathrm{~V} /+18 \mathrm{~V} /-5 \mathrm{~V} ; \mathrm{T}_{\text {case }}=25^{\circ} \mathrm{C} ; \mathrm{R}_{\mathrm{G}}=47 \Omega$ ).

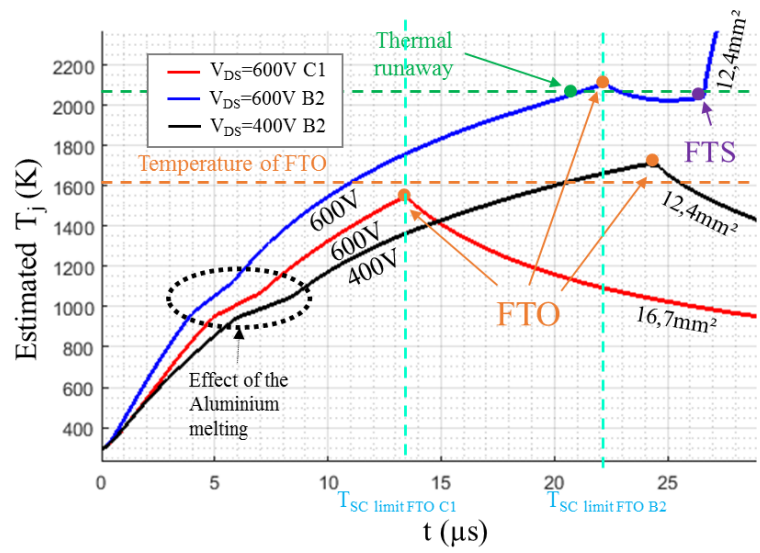

Fig. 7: Estimated junction temperature for devices $\mathrm{C} 1$ at $\mathrm{V}_{\mathrm{DS}}=600 \mathrm{~V}$ and $\mathrm{B} 2$ at $\mathrm{V}_{\mathrm{DS}}=600 \mathrm{~V} \& 400 \mathrm{~V}$.

However, three (A1, B2 and D1) of the tested devices presented the two failure modes in 
competition, as presented in Fig 6 for B2. Indeed, before the device failure in FTO mode (orange point) the drain leakage current had started to increase (green point). After the first failure, even if the device is turned-off, the thermally generated current is still self-heating the device leading to the second and permanent failure mode in FTS mode (purple point). This mechanism can be explained by looking at the estimated junction temperature in Fig. 7, for tests at $\mathrm{V}_{\mathrm{DS}}=600 \mathrm{~V} \& 400 \mathrm{~V}$. Indeed, the failure temperature in FTO mode is under $1700 \mathrm{~K}$ for the test at $400 \mathrm{~V}$. However, for the test at $600 \mathrm{~V}$, the FTO temperature is visible at about $2000 \mathrm{~K}$. It means that, to append, the failure mode in FTO needs a minimum time over a fixed temperature (1600$1700 \mathrm{~K})$. This time is named $\mathrm{T}_{\mathrm{SC}}$ limit FTO. Nevertheless, if the temperature is rising too fast, the thermal runaway can happen just before the $\mathrm{T}_{\mathrm{SC} \text { limit }}$ FTO leading first to a FTO and then to a FTS.

Finally, the device D1 has an unusual gradual gate damage as presented in Fig. 8. Indeed, step by step, the gate current increases resulting in a drop on the gate voltage leading to the SC drain current decrease. Each step are clearly identifiable in the waveforms. In the end, at $255 \mu \mathrm{s}$, the device is turned off. The physical explanation of this, step by step degradation could be caused by a gradual flow of the source top aluminium to the top gate finger as explained in the next section.
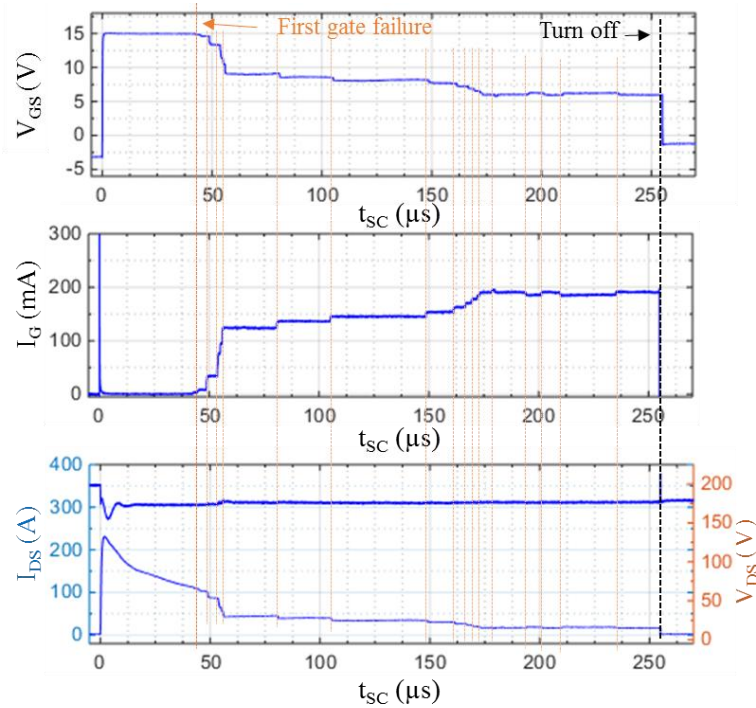

Fig. 8: Experimental waveforms during short-circuit D1. $\left(\mathrm{V}_{\mathrm{DS}}=200 \mathrm{~V} ; \mathrm{V}_{\text {buffer}(\text { on } / \text { off })}=+15 \mathrm{~V} /-3 \mathrm{~V} ; \mathrm{T}_{\text {case }}=\right.$ $25^{\circ} \mathrm{C} ; \mathrm{R}_{\mathrm{G}}=47 \Omega$ ).

\section{Failure analysis of the failure mode in FTO}

\subsection{Physical analysis}

As demonstrated in the previous section, the FTO mode is widely present in SiC MOSFET devices transiently or permanently. However, the physical failure mechanism is not well known today. Indeed, an investigation on the defected device D1, presented in Fig.8a, shows a metallic fusion area between the gate and the source on the aluminium top metal of the die, under the polyimide layer passivation. With a bias between the gate and the source, the LIT (Lock-In Thermography) images (Fig. 9b) shows a heat point proving the existence of an electrical path. An investigation conducted in [10] on the device B1 show the same kind mechanism. However, an investigation conducted in [11] on the device A2 shows cracks across the $\mathrm{SiO}_{2}$ dielectric around the Poly-Silicon gate in the die volume, with or without metal diffusion in it. These two results show that the failure mechanism could be caused either by the top aluminum flow through passivation or through gate oxide creating a resistive path. The results are consistent with the thermal model because the FTO mode appears after the fusion of the aluminum but owing to a delay which is assumed to be caused by its propagation time through the gate metal.

Analysis from physical investigation on devices in FTO are still in progress for the devices A2, C1 and D1.

In conclusion, the FTO mode seems to be caused by the liquid aluminium flowing to the gate metal. This mechanism is triggered by the important temperature inside the chip but the temperature has to remain under the temperature threshold which leads to thermal runaway in order to be stable and consolidated. 


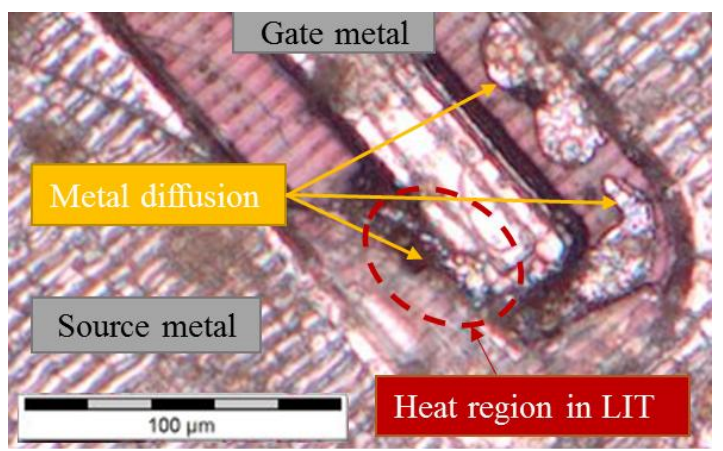

(a)

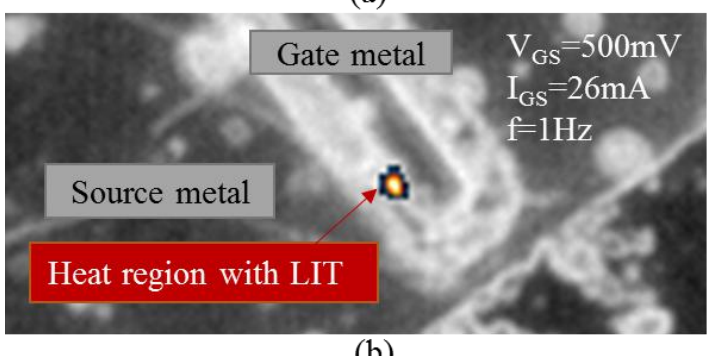

Fig. 9: Images of the damaged area on the device D1 (a) Optical microscope. (b) Lock-in thermography.

\subsection{Electrical characteristics}

II. Summary of the default gate-source resistance

As explained in section 3, the FTO mode is a shortcircuit between the gate and the source leading to the device turn-off. In order take advantage of this failure mode for safety operations, the gate source impedance and the body diode on-state performances must be characterized after FTO.

The measured gate source default resistance $\left(\mathrm{R}_{\mathrm{GS}}\right.$ Def) is summarised in table II. Two kind of FTO are

\begin{tabular}{|l|l|l|l|l|l|l|}
\hline Device & A2 & B1 & B2 & B3 & C1 & D1 \\
\hline $\begin{array}{l}\mathrm{R}_{\text {GS Def }}(\Omega) \\
(\text { Min/Max })\end{array}$ & $0.2 / 0.5$ & 0.8 & 0.6 & 0.2 & $18 / 20$ & 17 \\
\hline
\end{tabular}

identifiable: the ones with a low default resistance (lower than 1 $\Omega$ ) and the ones with a higher

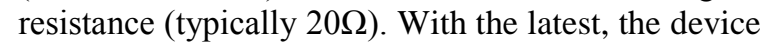
can still be turned-on if the current injected is sufficient. This particularity has to be well taken into account for future safety applications.

Despite the gate failure, the body diode of all the devices which failed in FTO is still usable as presented in Fig. 10. The body diode characteristic has been measured before and after the failure. It does not change for device $\mathrm{C} 1$. Intrinsic diode of device A2 presents a reduction of the forward voltage $\left(\mathrm{V}_{\mathrm{f}}\right)$. However, D1 presents an increase of $\mathrm{V}_{\mathrm{f}}$. Despite the small changes in the body diode characteristics, it is still functional which is essential for safety application with inductive load (majority of applications).

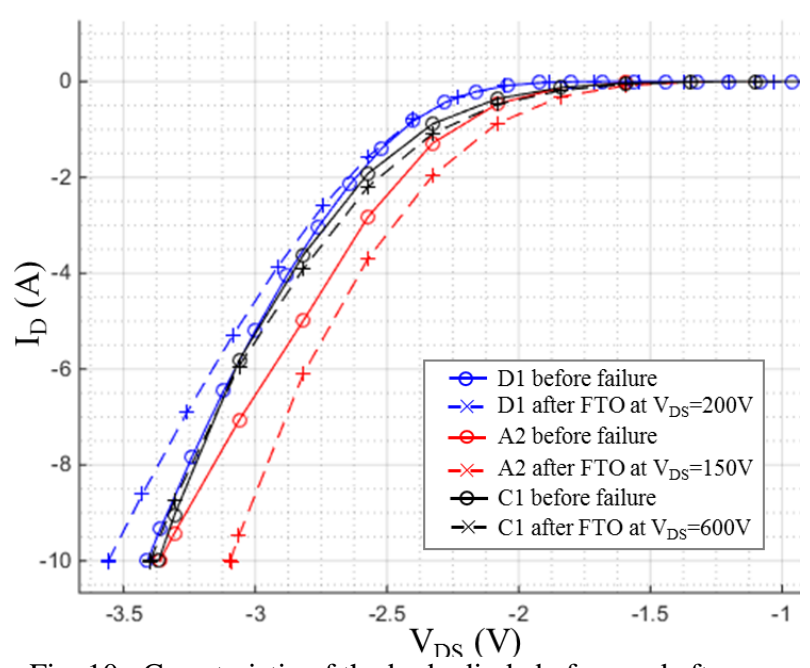

Fig. 10 : Caracteristic of the body diode before and after failure in FTO. Measure pulsed with an SMU Agilent.

$$
\mathrm{V}_{\mathrm{GS}}=0 \mathrm{~V}
$$

\section{Conclusion}

The results obtained confirm the existence of two failure modes (FTO and FTS) for seven devices from four manufacturers. However, each device has its own behaviour during the short-circuit and its own time before failure, at fixed voltage bias, depending on several parameters (chip size, doping, cells topology...). However, the $4 \mathrm{H}-\mathrm{SiC}$ particular thermal properties shift the temperature of thermal runaway of devices to a much higher level than what already observed with Si devices. Hence, new failure mechanisms are uncovered especially in the gate region which were not visible in $\mathrm{Si}$ technology. However, the studied failure mode in FTO appears at a temperature level close to the thermal runaway threshold and, once the failure temperature, is attained, the mechanism has a delay to append. This particular delay seems to depend on the paths available for the melted aluminium to reach the gate metal and, therefore, depend on the device internal structure. The device C1 and B2 internal structures seems to be the more appropriate to ensure the failsafe mode in FTO.

\section{Acknowledgement}


This research work received financial support from the French National Research Agency (ANR). Project name: HIT-TEMS managed by CNAM Paris and Satie lab.

\section{References}

[1]C. Chen et al., Microelectron. Reliab., vol. 55, no. 9-10, pp. 1708-1713, 2015.

[2]G. Romano et al., IEEE J. Emerg. Sel. Top. Power Electron. vol. 4, no. 3, pp. 978-987, 2016.

[3]Z. Dou, Thesis, Toulouse, INPT, 2011.

[4]F. Boige et al., ElectrIMACS, May 2017.

[5]S. Clemente, IEEE Trans. Power Electron., vol. 8, no. 4, pp. 337-341, 1993.

[6]J. E. Hatch, Aluminum: Properties and Physical Metallurgy. ASM International, 1984

[7]L. L. Snead et al., J. Nucl. Mater., vol. 371, no. 1, pp. 329377, Sep. 2007.

[8]F. Boige et al., Microelectron. Reliab., vol. 76-77, no. Supplement C, pp. 532-538, Sep. 2017.

[9]F.-C. Chiu, Advances in Materials Science and Engineering, vol. 2014, Article ID 578168

[10]D. Othman, Thesis, Paris Saclay, 2015.

[11]F. Boige et al., Microelectron. Reliab., vol. 76-77, no. Supplement C, pp. 500-506, Sep. 2017. 